\title{
Welcome Message from the Editorial Board Members: Introducing Journal of Cosmetology \& Trichology
}

\section{Vincenzo Nobile*}

Farcoderm s.r.l, Member of Complife Group, Via Mons Angelini, 21, 27028 San Martino Siccomario, Pavia, Italy.

*Corresponding author: Nobile V, Farcoderm s.r.l, Member of Complife Group, Via Mons Angelini, 21, 27028 San Martino Siccomario, Pavia, Italy, Tel: +39-0382-25504; E-mail: vincenzo.nobile@farcoderm.com

Received date: November 10, 2015; Accepted date: November 15, 2015; Published date: November 17, 2015

Copyright: ( 2015 Nobile V. This is an open-access article distributed under the terms of the Creative Commons Attribution License, which permits unrestricted use, distribution, and reproduction in any medium, provided the original author and source are credited.

\section{Welcome Message}

Dear readers,

On behalf of the editorial board members, it is with great pleasure, humility and honour that I welcome you to this inaugural first issue of Journal of Cosmetology \& Trichology, an international peer-reviewed journal published by OMICS International.

The journal has a strong emphasis on interdisciplinary issues reflecting the complexity of cosmetology and trichology. Today, cosmetology and trichology are a very broad research area involving different scientific disciplines ranging from chemistry, to basic sciences and from medicine to marketing. In each of this areas there are different journals dealing with very specific aspects intended for a limited audience. Similarly, interdisciplinary researches are often difficult to publish in specialized journals and part of the aim of Journal of Cosmetology \& Trichology is to capture high-quality works that doesn't clearly fall within the scope of other journals. This openminded stance toward the scope of the journal allows to overcome knowledge fragmentation and the integration between apparently different topics providing the reader with a global view on the topic of interest.

The protection of Consumers health and the protection of Consumer from misleading claims concerning efficacy and other characteristics of cosmetics are the central core of the worldwide regulatory framework. However, despite the increase of regulatory requirements a few of standards and guidelines exist. Robust scientific evidences should be given to support each claim made on cosmetic product, even if "product testing should not be only a regulatory concern but a prerequisite in order to ensure product safety and efficacy and to improve product credibility in the marketplace". The exchange of peer-reviewed global information with no boundaries and restrictions is then fundamental to improve the quality and competitiveness of cosmetic products in terms of good science applied to real life and Consumer protection.

The editorial board members and the peer-reviewing process will stimulate the opening of a constructive debate on safety and efficacy testing scientific requirements with the vision to fill the gap due to the lack of recognized protocols.

As editorial board member, I am delighted to be a part of this new interdisciplinary initiative, which I believe is exactly the type of platform needed to highlight and broaden cosmetology and trichology into widespread acceptance with the highest values of scientific integrity to which the Journal of Cosmetology \& Trichology is inspired.

Once more, on behalf of the editorial board members, I welcome you to this journal. We look forward to your submissions and to publish your manuscripts. 